\title{
Endometriose e sua relação com o câncer de ovário: uma revisão integrativa
}

\author{
Endometriosis and its relation to ovarian cancer: an integrative review \\ Endometriosis y su relación con el cáncer de ovario: una revisión integradora
}

Recebido: 22/09/2021 | Revisado: 29/09/2021 | Aceito: 30/09/2021 | Publicado: 03/10/2021

\author{
Tamyris Silva Alves \\ ORCID: https://orcid.org/0000-0003-0379-8006 \\ Universidade de Uberaba, Brasil \\ E-mail: tamyris.alves2015@gmail.com \\ Talita Silva Alves \\ ORCID:https://orcid.org/0000-0002-7602-1866 \\ Universidade de Uberaba, Brasil \\ E-mail:talitasilvaalves03@gmail.com \\ Igor Camargos Cunha Ferreira de Queiroz \\ ORCID:https://orcid.org/0000-0002-9516-6956 \\ Universidade de Uberaba, Brasil \\ E-mail: igorccqueiroz@gmail.com \\ Sabrina Marques Ferreira \\ ORCID:https://orcid.org/0000-0002-9035-6443 \\ Universidade de Uberaba, Brasil \\ E-mail: sabrinamf@edu.uniube.br \\ Júlia Oliveira Perez \\ ORCID:https://orcid.org/0000-0003-4919-5550 \\ Universidade de Uberaba, Brasil \\ E-mail: juliaoperez15@gmail.com \\ Paulo Adriano Ricardo Júnior \\ ORCID: https://orcid.org/0000-0002-8787-2519 \\ Universidade de Uberaba, Brasil \\ E-mail: paulo040497@hotmail.com \\ Heloise Paranaiba Almeida Drummond \\ ORCID:https://orcid.org/0000-0003-3206-0991 \\ Universidade de Uberaba, Brasil \\ E-mail: heloise_drummond_@hotmail.com \\ Douglas Reis Abdalla \\ ORCID:https://orcid.org/0000-0002-6971-1201 \\ Universidade de Uberaba, Brasil \\ E-mail: drabdalla@facthus.edu.br
}

\begin{abstract}
Resumo
A endometriose é uma reação inflamatória causada pela presença de tecido endometrial extrauterino que acomete mulheres em idade reprodutiva, e pode estar associada ao surgimento de patologias como os diversos tipos de câncer de ovário. O objetivo do presente trabalho foi encontrar na literatura evidências que associem a endometriose ao desenvolvimento de cânceres de ovário. Para a seleção de artigos conduzida em 6 etapas metodológicas: elaboração da questão norteadora, estabelecimento dos critérios de inclusão e exclusão dos artigos, leitura exploratória dos títulos e resumos dos artigos para a pré-seleção, leitura integral dos artigos, interpretação dos resultados e síntese seguida de apresentação dos resultados identificados. Foram encontradas 14 publicações entre os anos 2010 e 2020 , dos quais 4 $(28,57 \%)$ foram publicados na China, 4 (28,57\%) nos Estados Unidos, 3 (21,42\%) na Espanha, 2 (14,8\%) no Japão e $1(7,14 \%)$ na Romênia. Os tipos de estudos variaram entre relato de caso, observacional, comparativos e ensaios clínicos randomizados. Não houve prevalente uniformidade dos resultados encontrados. Os estudos analisados neste trabalho não demonstraram relação significativa entre o câncer de ovário e a endometriose, sendo necessárias mais investigações, mas apontam para opções mais eficientes de diagnósticos de tumores ovarianos em suas fases inicias, possibilitando um melhor prognóstico.
\end{abstract}

Palavras-chave: Endometriose; Câncer de ovário; Marcadores tumorais; Fatores de riscos.

\begin{abstract}
Endometriosis is an inflammatory reaction caused by the presence of extrauterine endometrial tissue that affects women of reproductive age, and may be associated with the development of pathologies such as several types of ovarian cancer. The aim of this study was to find in the literature evidence that associates endometriosis with the development of ovarian cancers. For the selection of articles conducted in 6 methodological steps: preparation of the guiding question, establishment of inclusion and exclusion criteria of articles, exploratory reading of titles and abstracts of articles for pre-selection, full reading of articles, interpretation of results and synthesis followed by
\end{abstract}


presentation of the results identified. Fourteen publications were found between the years 2010 and 2020, of which 4 $(28.57 \%)$ were published in China, $4(28.57 \%)$ in the United States, $3(21.42 \%)$ in Spain, 2 (14.8\%) in Japan, and 1 $(7.14 \%)$ in Romania. Study types ranged from case report, observational, comparative, and randomized clinical trials. There was no prevalent uniformity of the results found. The studies analyzed in this paper showed no significant relationship between ovarian cancer and endometriosis, and further investigations are needed, but point to more efficient options for diagnosing ovarian tumors in their early stages, enabling a better prognosis.

Keywords: Endometriosis; Ovarian cancer; Tumor markers; Risk factors.

\begin{abstract}
Resumen
La endometriosis es una reacción inflamatoria causada por la presencia de tejido endometrial extrauterino que afecta a las mujeres en edad reproductiva, y puede estar asociada a la aparición de patologías como varios tipos de cáncer de ovario. El objetivo del presente trabajo es encontrar en la literatura evidencias que asocien la endometriosis con el desarrollo de cánceres de ovario. Para la selección de los artículos se realizaron 6 pasos metodológicos: elaboración de la pregunta guía, establecimiento de los criterios de inclusión y exclusión de los artículos, lectura exploratoria de los títulos y resúmenes de los artículos para la preselección, lectura completa de los artículos, interpretación de los resultados y síntesis seguida de la presentación de los resultados identificados. Se encontraron 14 publicaciones entre los años 2010 y 2020, de las cuales 4 (28,57\%) se publicaron en China, 4 (28,57\%) en Estados Unidos, 3 (21,42\%) en España, $2(14,8 \%)$ en Japón y 1 (7,14\%) en Rumanía. Los tipos de estudios abarcaron desde informes de casos, observacionales, comparativos y ensayos clínicos aleatorios. No hubo uniformidad prevalente en los resultados encontrados. Los estudios analizados en este trabajo no mostraron una relación significativa entre el cáncer de ovario y la endometriosis, por lo que es necesario realizar más investigaciones, pero apuntan a opciones más eficaces para el diagnóstico de los tumores de ovario en sus fases iniciales, lo que permite un mejor pronóstico.
\end{abstract}

Palabras clave: Endometriosis; Cáncer de ovario; Marcadores tumorales; Factores de riesgo.

\title{
1. Introdução
}

A endometriose é uma doença ginecológica definida de acordo com a Sociedade Europeia de Reprodução Humana e Embriologia como a presença de tecido endometrial (glândula e/ou estroma) extrauterino, que provoca uma reação inflamatória e que acomete as mulheres em idade reprodutiva, ou seja, raramente aparece antes da menarca e após a menopausa (Mikhaleva et al., 2020). Essa patologia acomete, aproximadamente, 70 milhões de mulheres, sendo uma causa importante de internação ginecológica em países desenvolvidos (Oliveira et al., 2015). Há várias teorias que buscam explicar a origem da endometriose, todavia nenhuma explica todas as manifestações que a paciente com endometriose pode apresentar, como dor pélvica crônica, infertilidade, inflamação pélvica e aderências (Pearce et al., 2012).

De acordo com Salomé et al (2020), a faixa etária que apresentou maior número de casos no Brasil nos últimos cinco anos foi entre 40-49 anos, sendo mais prevalente em mulheres brancas. A região com maior número de internações por endometriose é a sudeste. Nos últimos cinco anos aconteceram 59.946 internações devido a endometriose, e a taxa de mortalidade foi de $0,15 \%$. A endometriose apresenta maior prevalência entre mulheres com maior nível de escolaridade, além de predominar em pacientes com união estável ou casadas. Em relação ao antecedente obstétrico, a maioria das pacientes eram nulíparas (Bellelis et al., 2010).

Dentre as teorias, têm-se a menstruação retrógrada, a qual, durante o período menstrual, ocorre refluxo de tecido endometrial através das tubas uterinas e a teoria da metaplasia celômica, a qual células mesoteliais se transformam em células endometrióticas, explicando o porquê de homens terem endometriose. Além dessas, há a teoria da haste endometrial, nela as células, que podem conter focos de endométrio, migram e se proliferam durante a embriogênese (Pearce et al., 2012).

O diagnóstico da endometriose será baseado na anamnese, no exame físico ginecológico e em exames complementares (ultrassom e ressonância nuclear magnética), porém, para confirmação, necessita-se do exame histológico por meio da laparoscopia, que é considerada padrão-ouro no diagnóstico. Ademais, a classificação da doença é da American Society of Reproductive Medicine, que irá graduar a patologia de acordo com a profundidade de invasão das lesões, com a bilateralidade e com o comprometimento dos anexos uterinos, bem como a densidade das aderências e o envolvimento do fundo de saco de Douglas (Oliveira et al., 2015). 
A endometriose é uma patologia considerada fator predisponente para o câncer de ovário, aumentando o risco em, aproximadamente, duas a três vezes, quando comparado com aquelas pacientes sem alterações (Olmos et al., 2020). Além disso, pode se transformar em forma atípica, em que há atipia celular, proliferação excessiva e até sofrer transformações malignas em 0,7-2,5\% dos casos (Mikhaleva et al., 2020). Alguns estudos relatam que essa atipia poderia ser uma lesão précancerígena, encontrada em $60 \%$ dos casos de endometriose com câncer de ovário, porém em apenas $2 \%$ dos casos sem câncer de ovário (Murakami et al., 2020). Pacientes com endometriose possuem relação com 15-50\% dos tumores ovarianos de células claras e linhagem endometrioide (Olmos et al., 2020).

São diversas as mutações encontradas que correlacionam a endometriose ao câncer de ovário, como mutação no gene ARID1A, que atua como supressor tumoral, no PIK3CA, KRAS e PI3K/AKT (Mikhaleva et al., 2020). Além disso, os marcadores tumorais, por exemplo, CA19-9 e CA-125 são importantes elementos para investigação de ambas as patologias; na endometriose, esse último é essencial para a realização de diagnóstico, quando a concentração é maior que 30UI/mL, e no câncer de ovário para seguimento, após diagnosticado o tumor (Mikhaleva et al., 2020).

O estresse oxidativo é outro item que permite associar a endometriose e o câncer de ovário, pois, quando enzimas responsáveis pela ação antioxidantes estão sobrecarregadas, o estresse oxidativo ocasiona danos a componentes essenciais, como lipídeos, proteínas e até mesmo o DNA, aumentando assim o risco de doenças, como as duas supracitadas (Alexandre et al., 2019). Ainda de acordo com este autor, a epigenética, termo associado a mudanças hereditárias na função gênica e que não podem ser explicadas por alterações na sequência do DNA, é utilizada para explicar a fisiopatologia da endometriose e da carcinogênese, visto que reprime os genes supressores tumorais e ativa os oncogenes. Um dos mecanismos da epigenética é a expressão de RNAs não codificadores, incluindo micro-RNAs (miRNA), envolvidos no processo de desenvolvimento, diferenciação, apoptose e proliferação e há estudos que acreditam na relação desses miRNA com o desenvolvimento de endometriose e do câncer de ovário associado a endometriose.

Apesar da Sociedade Mundial de Endometriose apoiar que o risco relativo e absoluto de câncer de ovário em mulheres com endometriose ser muito baixo e a triagem de rotina para câncer de ovário não ser recomendado, como descrito por Murakami et al (2020), há diversas alterações genéticas e moleculares, além do estresse oxidativo que associam as duas patologias, portanto é propício maior atenção ao assunto.

Desta forma, o objetivo do presente trabalho foi encontrar na literatura evidências que associem a endometriose ao desenvolvimento de cânceres de ovário.

\section{Metodologia}

No presente estudo, foi conduzida uma revisão integrativa, a qual consiste em uma pesquisa que permite, acerca das evidências encontradas, a avaliação, a síntese e o conhecimento sobre determinado fenômeno, assim como busca produzir uma visão macroscópica de terminologias complexas, de teorias ou de problemas de saúde relevantes a partir de trabalhos préexistentes, o que possibilita, dessa forma, a proposição de intervenção (Galvão et al., 2004; Whittemore; Knafl, 2005).

Para a escolha dos artigos, foi definido 6 etapas metodológicas: 1. elaboração de questão norteadora ou hipótese de pesquisa - isto é, identificou-se o problema, apresentou-se o mecanismo de busca e os descritores ou palavras-chave; 2. estabelecimento de critérios inclusivos e exclusivos para os artigos selecionados para a composição da amostra; 3. leitura exploratória dos títulos e dos resumos dos artigos, com o propósito de pré-seleção; 4. leitura analítica dos artigos, com o intuito de compilar, de analisar e de categorizar as informações; 5. interpretação dos resultados; 6. síntese seguida da apresentação dos resultados identificados, que permeiam a questão norteadora (De Sousa et al., 2011). 
Portanto, neste estudo, optou-se por realizar a busca sobre os conceitos: endometriose, câncer de ovário, marcadores tumorais, fatores de riscos. A partir desses conceitos estabelecidos, definiu-se a questão norteadora: A endometriose pode sofrer transformação maligna, tornando-se câncer de ovário e/ou aumentar o risco de câncer de ovário?

Após a formulação da questão a ser pesquisada, realizou-se um levantamento bibliográfico na plataforma PubMed. O levantamento da pesquisa ocorreu entre junho e julho de 2021. Já a seleção dos textos procedeu com as buscas na plataforma utilizando os filtros nela disponíveis para textos publicados entre 2010 e 2021. Para a seleção das publicações, adotaram-se os seguintes critérios de inclusão: artigos científicos, publicados no idioma Inglês, entre os anos de 2010 e 2020, disponíveis online e gratuitamente na íntegra. Somado a isso, foram excluídos os artigos sem resumo na base de dados ou que estavam incompletos, assim como também se excluiu os editoriais, as cartas ao editor, os estudos reflexivos, as revisões sistemáticas ou integrativas de literatura.

Ademais, subsequente à definição da questão norteadora, à localização e à seleção dos artigos, identificou-se 1.000 publicações potencialmente elegíveis para serem incluídas nesta revisão. Em seguida, com a aplicação dos critérios inclusivos e exclusivos, a amostragem foi composta por 90 publicações; analisaram-se os resumos de 26 registros, a fim de verificar se atendiam aos critérios de elegibilidade e se respondiam à pergunta norteadora desta revisão. Dessa forma, excluiu-se 12 registros e, apenas, 14 foram analisados na íntegra com a finalidade de confirmar a elegibilidade, para a síntese quantitativa e para a análise dos dados, conforme o fluxograma de seleção (Figura 1).

Figura 1: Fluxograma de seleção dos artigos.

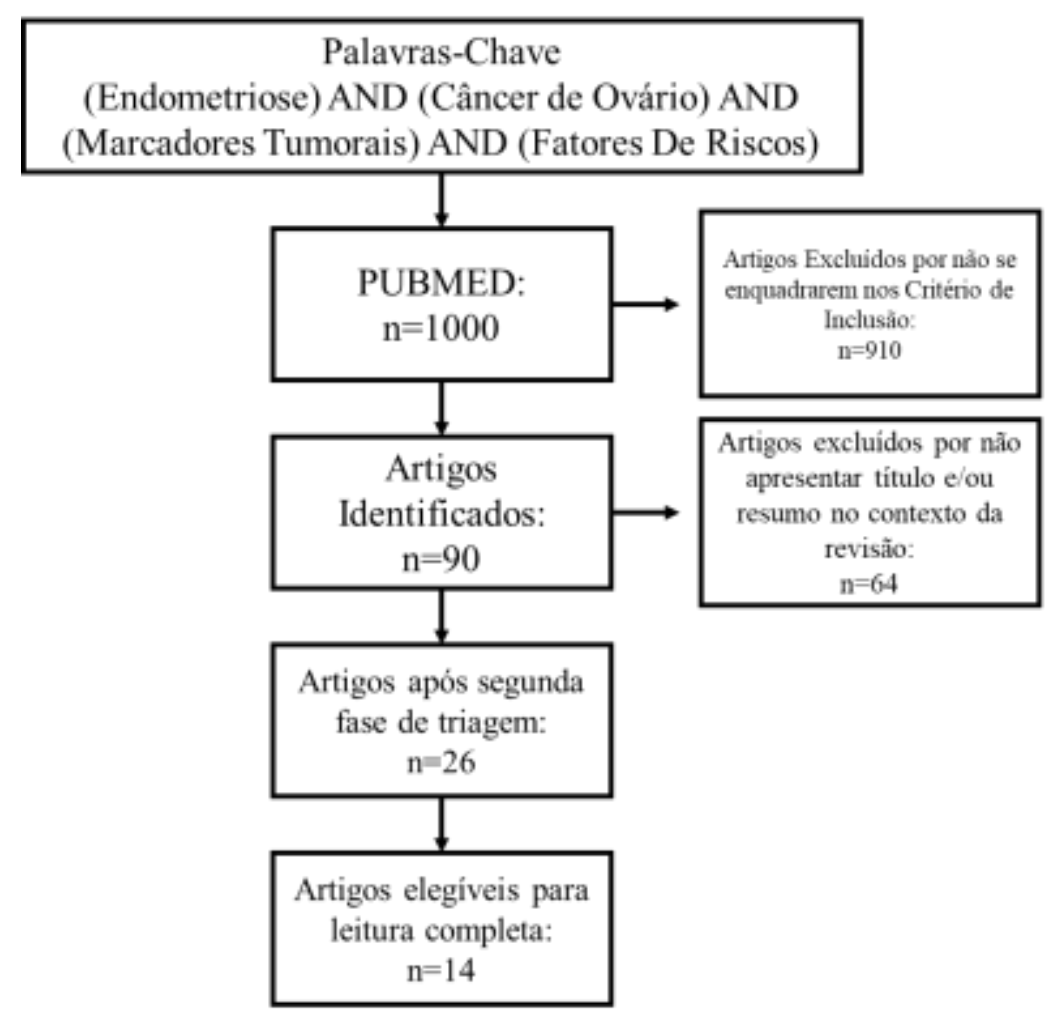

Fonte: Autores (2021).

\section{Resultados e Discussão}

No espaço de tempo delimitado para a realização deste estudo (2011-2020) foram encontradas e analisadas 14 publicações, sumarizadas na tabela 1. Em 2011, 2012, 2013 e 2018 foram publicados 1 artigos $(7,14 \%)$ em cada ano 
respectivamente. Nos anos de 2015 consta 2 publicações (14,28\%). Já nos anos de 2019 e 2020 foram publicados 3 (21,42\%) e $5(35,7 \%)$ artigos respectivamente.

De acordo com a metodologia dos trabalhos selecionados os tipos de estudo eram observacionais, comparativos, relato de casos, ensaio clínico randomizado que levantaram hipóteses de relação entre a endometriose e o câncer de ovário.

As publicações resultaram de diferentes revistas: Journal Gynecology Oncology. Journal of Ovarian Research, BMC Women's Health, Medicine, Journal of the Society of Laparoscopic \& Robotic Surgeons, Gynecology and Obstetric Investigation, Romanian Journal of Morphology \& Embryology, Journal of Assisted Reproduction and Genetics, Clinical Cancer Research, International Journal of Clinical and Experimental Pathology, World Journal of Surgical Oncology e Plos One. Analisando os locais de estudo 4 artigos foram realizados na China (28,57\%), outros 4 artigos desenvolvidos no Estados Unidos (28,57\%), 3 realizados na Espanha (21,42\%), 2 artigos no Japão (14,28\%) e foi publicado 1 artigo na Romênia $(7,14 \%)$.

Tabela 1 - Sumarização das publicações selecionadas para compor a revisão integrativa.

\begin{tabular}{|c|c|c|c|c|}
\hline $\mathbf{N}^{\mathbf{o}}$ & $\begin{array}{l}\text { Pesquisador } \\
\text { es }\end{array}$ & Ano & $\begin{array}{l}\text { Tipo de } \\
\text { Estudo }\end{array}$ & Objetivos do estudo \\
\hline 1 & Meng et al & 2011 & $\begin{array}{l}\text { Observacional } \\
\text { prospectivo }\end{array}$ & $\begin{array}{l}\text { Investigar uma possível associação entre a endometriose e o câncer de } \\
\text { ovário a partir do estudo da expressão de biomarcadores conhecidos por } \\
\text { influenciar a patogênese dessas doenças. }\end{array}$ \\
\hline 2 & Xiao et al & 2012 & $\begin{array}{l}\text { Estudo } \\
\text { comparativo }\end{array}$ & $\begin{array}{l}\text { Examinar se a endometriose benigna já acumulou alterações moleculares } \\
\text { que frequentemente são observadas na endometriose atípica e carcinoma } \\
\text { de células claras, analisando a expressão de BAF250a, e proteínas como } \\
\text { HNF-1b, ER, PR e P53. }\end{array}$ \\
\hline 3 & $\begin{array}{l}\text { Suryawanshi } \\
\quad \text { et al }\end{array}$ & 2013 & $\begin{array}{l}\text { Exploratório de } \\
\text { duas etapas }\end{array}$ & $\begin{array}{c}\text { Analisar a utilização de miRNAs plasmáticos como possíveis } \\
\text { biomarcadores a serem utilizados para a diferenciação entre pacientes } \\
\text { com endometriose, casos de cânceres epiteliais do ovário e pacientes } \\
\text { saudáveis }\end{array}$ \\
\hline 4 & Lee et al & 2015 & $\begin{array}{c}\text { Estudo } \\
\text { observacional }\end{array}$ & $\begin{array}{l}\text { Testar o impacto dos critérios diagnósticos de endometriose no cálculo } \\
\text { de risco de câncer epitelial de ovário. }\end{array}$ \\
\hline 5 & Acién et al & 2015 & Observacional & $\begin{array}{c}\text { Determinar a prevalência da endometriose em todos os casos de cânceres } \\
\text { epiteliais do ovário confirmados histopatologicamente operados dos } \\
\text { últimos } 20 \text { anos, assim como analisar a relação entre diferentes tipos } \\
\text { histológicos e endometriose. }\end{array}$ \\
\hline 6 & Zhang et al & 2018 & Observacional & $\begin{array}{c}\text { Identificar se a endometriose associada a EBOT (EAEBOT) } \\
\text { representava uma doença heterogênea, distinta de outras formas de } \\
\text { tumores ovarianos endometrioides borderline (EBOTs) em aspectos } \\
\text { clínicos, patológicos e prognóstico. }\end{array}$ \\
\hline 7 & Sevilla et al & 2019 & Observacional & $\begin{array}{l}\text { O objetivo do estudo foi investigar a possível existência de uma lesão } \\
\text { histológica precursora específica para câncer de ovário associado à } \\
\text { endometriose no espectro da endometriose, analisando o grau de } \\
\text { apresentação das duas condições histológicas. }\end{array}$ \\
\hline 8 & Nezhat et al & 2019 & Relato de caso & $\begin{array}{l}\text { Relatar um caso de rabdomiossarcoma agressivo que surge em } \\
\text { associação com endometriose extensa e postulamos que pode ter surgido } \\
\text { do crescimento excessivo do estroma em um adenosarcoma sutil. }\end{array}$ \\
\hline 9 & Vilches et al & 2019 & Relato de caso & $\begin{array}{c}\text { Relatar um caso de adenosarcoma extra-uterino que é uma patologia de } \\
\text { baixa incidência. }\end{array}$ \\
\hline
\end{tabular}




\begin{tabular}{|c|c|c|c|c|}
\hline 10 & Kwok et al & 2020 & Observacional & $\begin{array}{c}\text { O presente estudo investigou endometrioma ovariano (OEM) a fim de } \\
\text { determinar a distribuição das lesões características da endometriose } \\
\text { infiltrante profunda (DIE) associado ao OEM. }\end{array}$ \\
\hline 11 & Chao et al & 2020 & Observacional & $\begin{array}{c}\text { Fornecer uma referência para a prevenção, diagnóstico e tratamento de } \\
\text { tais condições através da análise retrospectiva das características clinico- } \\
\text { patológicas da massa pélvica em pacientes receberam histerectomia por } \\
\text { doenças benignas como endometriose e adenomiose. }\end{array}$ \\
\hline 12 & Penciu et al & 2020 & Retrospectivo & $\begin{array}{c}\text { Determinar se há alguma associação entre endometriose ovariana e } \\
\text { câncer de ovário concomitantes, assim como determinar se há diferenças } \\
\text { entre endometrioses associadas com diferentes tipos morfológicos de } \\
\text { câncer de ovário. }\end{array}$ \\
\hline 13 & Ota et al & 2020 & Relato de Caso & $\begin{array}{c}\text { Relatar um caso raro de carcinoma endometrioide decorrente de DIE no } \\
\text { ligamento uterossacro } 6 \text { anos após salpingo-ooforectomia bilateral } \\
\text { devido à ocorrência de tumor endometrioide proliferativo atípico do } \\
\text { ovário. }\end{array}$ \\
\hline 14 & $\begin{array}{c}\text { Okamoto et } \\
a l\end{array}$ & 2020 & $\begin{array}{c}\text { Observacional } \\
\text { Prospectivo }\end{array}$ & $\begin{array}{l}\text { Utilizar a hibridização genômica comparativa de alta resolução para } \\
\text { identificar alterações no número de cópias somáticas associadas às } \\
\text { características clínicas do adenocarcinoma de células claras do ovário. }\end{array}$ \\
\hline
\end{tabular}

Fonte: Autores.

No trabalho de Meng (2011), foi investigado uma possível associação entre a endometriose e o câncer de ovário a partir do estudo da expressão de biomarcadores conhecidos por influenciar a patogênese dessas doenças. Esses biomarcadores incluem fator de crescimento transformador (TGF)- $\beta 1$, cicloxigenase (COX)-2, fator de crescimento vascular endotelial (VEGF), receptor de estrogênio (ER)-1 $\alpha$, receptor de prolactina (PR), receptor de aromatase (AR) e aromatase. Para tanto, dois grupos de mulheres de idade entre 25-45 anos foram utilizadas na pesquisa. O primeiro grupo consistiu de 7 mulheres com endometriose que se submeteram a cirurgia por causa de dor pélvica, e tiverem cistos ovarianos retirados por laparoscopia. O grupo controle consistiu de 7 mulheres saudáveis, não grávidas, que fizeram cirurgia de apendicite e tiveram a excisão de peritônio normal. Foram analisadas as expressões gênicas dos marcadores TGF- $\beta 1$, COX-2, VEGF, ER-1 $\alpha$, PR, AR e aromatase. Foi encontrado que tecidos de endometriose ovariana e células de câncer ovariano epitelial (EOC, do inglês epithelial ovarian cancer) exibiram um aumento na expressão de níveis basais de TGF- $\beta 1$, COX-2, VEGF, ER-1 $\alpha$, AR e aromatase, e diminuição na expressão dos níveis basais de PR quando comparados ao peritônio normal. O padrão similar de expressão foi observado tanto em tecidos ovarianos com endometriose quanto em células de EOC pode indicar um mecanismo molecular comum entre as duas doenças. Assim, utilizando biomarcadores que ligam a inflamação, a endometriose e o câncer de ovário foi demonstrado uma associação entre as duas doenças em nível molecular. Devido ao pequeno valor de amostragem, a associação entre a endometriose e o câncer de ovário deve ser analisada mais profundamente.

No projeto de Suryawanshi et al (2013), foi analisada a utilização de miRNAs plasmáticos como possíveis biomarcadores a serem utilizados para a diferenciação entre pacientes com endometriose e EAOC (câncer ovariano associado à endometriose, do inglês endometriosis-associated ovarian cancer) de pacientes saudáveis. A amostra do estudo consistiu em pacientes do sexo feminino com endometriose $(n=33)$, EAOC $(n=14$, dos quais seis eram do tipo endometrioide, sete eram tumores de células clara e um possui uma mistura histológica de endometrioide/células claras) e câncer ovariano seroso ( $\mathrm{n}=$ 21). O grupo controle foi composto por amostras de plasma de mulheres saudáveis $(\mathrm{n}=20)$ comprado da Innovative Research Labs (Seattle, WA). Das 88 amostras de plasma coletadas, 20 eram aleatoriamente selecionadas para a fase inicial de descoberta (grupo controle, $\mathrm{n}=6$; endometriose, $\mathrm{n}=7$; EAOC, $\mathrm{n}=7$ dos quais quatro eram endometrioide e três eram tumores de células claras). Na fase de descoberta inicial, miRNAs extraídos de 20 amostras de plasma foram utilizados para quantificar 
um total de 1113 miRNAs por RT-qPCR. A aplicação de LDA (análise de discriminantes lineares) revelou três miRNAs plasmáticos, miR- 16, 191 e 195, todos regulados para cima na endometriose, que poderiam diferenciar indivíduos saudáveis de casos de endometriose com 88\% de sensibilidade (SN), e 60\% de especificidade (SP). Uma combinação de miR-16, 21 e 191 podem diferenciar saudáveis de casos de EOAC com 86\% de SN e 85\% de SP, enquanto miR-21, 362-5p e 1274 podem diferenciar endometriose de EAOC com 57\% de SN 91\% de SP. miR-21, 191 e 1975 podem distinguir juntamente EAOC de câncer ovariano seroso (SOC) com 86\% de SN e 79\% de SP. A assinatura de expressão de miR-16, 191 e 4284 poderia ser utilizada para discernir entre indivíduos saudáveis de pacientes com SOC com 90\% de SN e 55\% de SP, enquanto que miR362-5p, 628-3p e 1915 podem diferenciar endometriose de SOC com 90\% d SN e 73\% de SP. Uma tendência geral da expressão elevada de miRNA também foi notada no grupo controle em relação aos casos de endometriose e EAOC, mas não com os casos de SOC, sugerindo que esses miRNAs podem servir como novos biomarcadores, que refletem a progressão patológica de benigna para lesão precursora para EAOC totalmente desenvolvido. Dessa forma, os miRNAs identificados no estudo representam biomarcadores promissores da progressão de endometriose até EAOC, e podem ser úteis para melhorar os resultados clínicos de pacientes com câncer de ovário.

Segundo pesquisa realizada por Lee et al. (2015), buscaram testar o impacto dos critérios diagnósticos de endometriose no cálculo de risco de câncer epitelial de ovário (EOC). Para isso, foi feito um estudo de coorte histórico nacional de mulheres entre 1996 e 2010 com 239.385 mulheres da população de Taiwan, com idade entre 20 e 51 anos, sendo que as mulheres com endometriose deveriam ser detectadas inicialmente entre o ano de 2000 e 2010. Já as que não possuíam qualquer endometriose foram classificadas como controle. Do total de indivíduos participantes, 348 tiveram EOC entre $2001 \mathrm{e}$ 2010. Com relação a taxa de incidência de EOC das mulheres com endometriose, ocorreu grande variação, variando de 1,9 por 10.000 pessoas-ano em endometriose recordada a 18,7 por 10.000 pessoas-ano em endometrioma ovariano comprovado por tecido. Já a taxa de incidência de EOC das mulheres sem endometriose variou de 0,8 a 0,9 por 10.000 pessoas-ano e esse dado foi próximo ao índice de incidência padrão de idade (do EOC após ajuste de idade em Taiwan $(0,8-0,9$ por 10.000 pessoasano). Esses resultados sugeriram que mulheres com endometriose tiveram um intervalo significativamente menor para desenvolver EOC, em comparação com o intervalo mais longo de mulheres sem endometriose. Uma vez que o maior risco de EOC pode ser identificado no primeiro ano de acompanhamento, levantou-se a questão de que o risco de EOC em mulheres com endometriose pode ser tendencioso pela vigilância. Além disso, descobriram que as mulheres com endometriose tinham um risco consistente e persiste persistentemente maior de EOC do que as mulheres sem endometriose, independentemente dos critérios usados. Assim, reconfirmaram uma associação previamente relatada entre endometriose e risco aumentado de EOC e que o risco de EOC em mulheres com endometriose pode ser maior.

De acordo com o trabalho de Acién et al. (2015), os autores visaram determinar a prevalência da endometriose em todos os casos de EOC (câncer epitelial de ovário, do inglês epithelial ovarian cancer) ambos invasivo e borderline, assim como analisar a relação entre diferentes tipos histológicos de EOC e endometriose, avaliando a possibilidade de progressão patológica nos pacientes - a relação entre essas patologias e o carcinoma endometrial também foi considerada. Para isso, foi elaborado um estudo coorte observacional, no qual a amostra consistiu em 192 pacientes do sexo feminino confirmadas histopatologicamente para EOC e operadas, dentro dos últimos 20 anos. Dentro desses casos, 30 mulheres apresentaram endometriose atípica e 17 endometriose positiva para p53. A partir disso, analisou e associou-se a endometriose e os carcinomas endometriais, os subtipos histológicos, o estágio tumoral e as características clínicas e patológicas, assim como a taxa de sobrevivência. Como resultado, obteve-se: 20 casos de EOC (10,4\%) tinham endometriose (12,7\% borderline e 9,3\% casos invasivos); endometriose associada com EOC seroso ou mucinoso foi observado em 2,2 e 2,7\% dos casos, respectivamente - essa associação também foi encontrada em EOC do tipo endometrioide (50\%) e de célula clara (23\%) -; a idade, a paridade e o estágio do tumor foram baixos nos pacientes com endometriose associada a EOC; todos os casos de 
associação era do tipo I (na classificação de Kurman e Shih) e demonstraram melhores resultados na taxa de recuperação/sobrevivência e carcinoma endometrial foi mais frequentemente associado a EOC endometrioide (25\%). Em suma, há significativa associação entre endometriose - incluindo as formas atípicas - e carcinomas do tipo endometrioide e de célula clara, mas não com outros histotipos de EOC. A presença de endometriose em EOC sugere um melhor prognóstico e um estágio intermediário dentro da progressão endometriose-carcinoma.

Zhang et al. (2018), em análise realizada, buscaram identificar se a endometriose associada a EBOT (EAEBOT) representava uma doença heterogênea, distinta de outras formas de tumores ovarianos endometrioides borderlines (EBOTs) em aspectos clínicos, patológicos e prognóstico. Para isso foi elaborado um estudo coorte retrospectivo de 1995 a 2015 a partir de revisão de prontuários médicos de 52 pacientes. As variáveis consideradas foram: idade, estado de fertilidade, gravidez, sintomas, estado menopausal, níveis de CA-125, data da cirurgia, diâmetro máximo do tumor, ruptura do cisto, estágio FIGO, abordagem cirúrgica (laparoscopia ou laparotomia), estadiamento, quimioterapia, concomitante a endometriose, data de progressão ou recidiva da doença. As pacientes foram divididas em dois grupos, de acordo com a detecção de EBOT resultante de endometriose ou não. Especificamente, a EAEBOT foi definida como segue: presença de EBOT e endometriose no mesmo ovário, presença de endometriose em um ovário e de EBOT no ovário contralateral ou presença de EBOT e endometriose extraovariana. Das 52 pacientes participantes do projeto, 19 (36,5\%) foram associadas à endometriose e alocadas para um grupo e o restante foi para outro, sem endometriose. A faixa etária do diagnóstico foi entre 23 e 81 anos sendo que 12 (23,1\%) estavam na menopausa, $15(29,4 \%)$ tinham pelo menos 3 gravidez e 24 (46,2\%) eram nulíparas. Quanto ao estágio FIGO foi de 49(94,2\%) para estágio I, 2 para o estágio II, 1 para o estágio III, e 0 para o estágio IV. Ainda, 47 (90,4\%) pacientes estavam com tumores, $12(23,1 \%)$ receberam quimioterapia após a cirurgia primária e 8(15,4\%) tinham neoplasia endometrial intraepitelial (EIN) concomitante e 11(21,2\%) tinham câncer endometrial (CE). O sintoma mais comum para ambos os grupos era massa pélvica, seguida de sangramento vaginal. Além disso, 9 (17,3\%) progressões ou recidivas de doenças foram observadas e teve apenas 2 casos de transformação maligna. Já em relação aos sintomas, nível de CA125 e tamanho do tumor não houve diferenças estatisticamente entre os dois grupos. Portanto, após as analises, concluiu que não houve diferenças significativas entre a EAEBOT e nãoEAEBOT em muitos aspectos principalmente em características clinico patológicas e prognóstico, considerando então, a EAEBOT como uma entidade similar à nãoEAEBOT e pacientes com a EAEBOT eram mais propensas a serem mais jovens e na pré-menopausa.

No estudo de Sevilla et al. (2019), buscaram investigar a possível existência de um precursor específico para câncer de ovário associado à endometriose (EAOC) no espectro da endometriose, analisando o grau de apresentação das duas condições histológicas incluídas na endometriose atípica (atipia celular e atipia ou hiperplasia arquitetural). Para isso, foi elaborado o delineamento prospectivo e observacional entre janeiro de 2014 a abril de 2017 com pacientes submetidas a procedimento cirúrgico, em que 266 tiveram a confirmação histológica do diagnóstico de endometriose, câncer ovariano, ou EAOC. Pacientes sem confirmação histológica, por nenhum dos dois patologistas, de endometriose ou câncer de ovário foram excluídos do estudo. Em 185 pacientes com endometriose, resultados característicos da endometriose atípica foram relatados pelos patologistas em 23 casos (12,43\%), dos quais 14 (60,86\%) tinham apenas endometriose (ou seja, sem neoplasia associada) e o outros 9 (39,13\%) tinham EAOC. Ainda, dos 23 casos de endometriose atípica constatou que $11(47,8 \%)$ pacientes tinham endometriose com citologia atípica (ECA) e $12(52,2 \%)$ tinham endometriose com atipia arquitetônica (EAA). A ECA foi encontrada principalmente em pacientes sem neoplasia $(71,4 \%)$ e a EAA foi observada em pacientes com câncer de ovário $(88,9 \%)$ ( $\mathrm{p}=0,009)$. Ademais, entre os 9 casos de EAOC com endometriose atípica, 8 eram EAA e 1 era ECA. Ao analisar a expressão de coloração para $\mathrm{COX} 2$, marcador inflamatório, encontrou-se maior expressão na endometriose típica comparada com a atípica $(\mathrm{p}<0,001)$ e quando comparado apenas as formas atípicas de endometriose, a COX2 foi positiva em $80 \%$ dos casos de ECA e $20 \%$ de EAA ( $(\mathrm{p}=0,089)$. Ainda, o marcador de proliferação celular Ki-67 foi maior em pacientes com 
endometriose atípica do que naqueles com endometriose típica $(\mathrm{p}<0,001)$ e foi maior em mulheres com EAA comparado aquelas com ECA ( $\mathrm{p}=0,004)$. Em relação ao marcador da mutação do gene ARID1A, o BAF250a, a perda de sua expressão foi de 9,1 para ECA versus $40 \%$ para EAA (p=0,149). Por último, em relação ao tipo histológico, o subtipo seroso foi positivo em 53,1\% dos casos não EAOC e 15,4\% nos pacientes com EAOC. Além disso, 23,1\% dos casos de EAOC foram adenocarcinoma de células claras (CCA) e 42,3\% eram adenocarcinoma endometrioide (EA), em comparação com 6,2\% e 14,8\% respectivamente, dos casos observados em não EAOC ( $p<0,001)$. Logo, os resultados sugerem que a endometriose atípica é uma lesão pré-maligna para o câncer de ovário, como ocorre no câncer endometrial. Possivelmente há uma associação significativa de EAA com EAOC, que também exibe uma atividade proliferativa mais pronunciada do que no caso da ECA, como refletido por um índice Ki-67 mais alto, além de uma maior perda da expressão BAF250a. Por fim, um patologista que observa a endometriose também deve olhar além do diagnóstico desta condição para examinar o espécime e identificar presença de lesões histológicas que poderia ser descrita como endometriose atípica, particularmente EAA, e, se encontrada, cuidadosamente descartar a presença de malignidade no resto dos espécimes histológicos.

Conforme o estudo conduzido por Nezhat et al (2019), relataram um caso de rabdomiossarcoma agressivo que surge em associação com endometriose extensa, visto que rabdomiossarcoma extrauterino de origem ginecológica foi raramente relatado, embora sua relação com a endometriose subjacente nem sempre seja discutida. O caso relata histórico de endometriose, com recidiva após 3 meses de tratamento cirúrgico, que devido ao rápido crescimento e sintomatologia intensa realizou-se laparoscopia diagnostica, no qual a patologia final das amostras revelou rabdomiossarcoma nas amostras ovarianas e peritoneais. As colorações imunohistoquímicas mostraram que as células fusiformes eram positivas para miogenina e PAX-7, consistente com diferenciação rabdomioblástica. Os achados morfológicos e imunofenotípicos gerais foram interpretados como sarcoma maligno com diferenciação rabdomioblástica, consistente com rabdomiossarcoma ovariano, tipo embrionário. Após esse achado, os espécimes da cirurgia anterior do paciente foram revisados uma segunda vez. As colorações imunohistoquímicas foram realizadas retroativamente em suas lâminas anteriores, que também demonstraram miogenina positiva e PAX-7 em algumas tiras de estroma mais pálido e levemente atípico adjacente à endometriose convencional. Esses focos em retrospecto eram consistentes com diferenciação rabdomiossarcomatosa focal. Espaços glandulares císticos raros com balão / condensação de estroma do tipo endometrial de aparência branda foram observados no mesmo slide, sugerindo possível adenosarcoma. Os achados cumulativos foram favorecidos por representar um crescimento excessivo do estroma extenso com diferenciação rabdomiossarcomatosa em um adenosarcoma, decorrente de um endometrioma de fundo. Em posterior tratamento cirúrgico paliativo a patologia final demonstrou sarcoma de alto grau com diferenciação rabdomioblástica e condroblástica no mioma uterino e serosa, segmento posterior do útero inferior, ovários bilaterais e nos linfonodos aórticos e ilíacos externos direitos. Assim, foi concluído que há uma prevalência maior de câncer de ovário entre mulheres com histórico de endometriose em comparação com a população em geral. Endometriose também pode mostrar transformação maligna em neoplasias estromais mistas e epiteliais ou exclusivamente estromais, incluindo sarcoma estromal endometrial, adenocarcinoma de células claras e adenosarcoma com componentes heterólogos de rabdomiossarcoma e adenosarcoma ovariano com rabdomiossarcoma.

Jimenez et al (2019), relataram um caso de adenosarcoma com baixo grau de localização extrauterina e com acometimento da parede uterina, ovário e vesical, o objetivo do artigo foi relatar um caso de adenosarcoma extrauterino que é uma patologia de baixa incidência, sendo que se acredita que os tumores extrauterinos surjam de depósito de endometriose. $\mathrm{O}$ caso relatado consiste em histórico de endometriose profunda, em acompanhamento há 3 anos. USTV apresentava cisto de 18 $\mathrm{mm}$ de aparência de endometriose; adjacente à formação tubular heterogênea do ovário de $54 \times 23 \mathrm{~mm}$. Com marcadores tumorais elevados CA 125253.7. Realizou-se abordagem cirúrgica visualizando múltiplos implantes de endometriose peritoneal. Foram realizadas três biópsias intraoperatórias (biópsia de lesão anexial esquerda, tecido vesical e lesão para- 
anexial esquerda) devido à natureza incomum das lesões e sua infiltração, todas relatadas como endometriose. Realizou-se a cirurgia e o resultado da peça cirúrgica foi: útero com endométrio inativo, adenomiose extensa, mioma intramural de $3 \mathrm{~cm}$ e adenosarcoma de baixo grau. Ovário esquerdo com um mioma e adenosarcoma de baixo grau. Fragmento da parede da bexiga com infiltração de adenosarcoma de baixo grau. Amostras de adenosarcoma com receptor de estrogênio positivo 100\%, receptor de progesterona positivo $100 \%$ e CD10 positivo. Assim concluímos que adenosarcoma mülleriano extrauterino é raro, mas deve ser considerado em uma paciente com características clínicas atípicas ou patologia pré-operatória, especialmente em uma paciente com história de endometriose.

De acordo com o elegante estudo de Kwoket al. (2020), buscaram avaliar a distribuição das lesões da endometriose profunda infiltrante (DIE) associada ao endometrioma ovariano (OEM). Com isso, foi elaborado um delineamento que entre junho de 2008 e junho de 2016 foram selecionados 304 pacientes do sexo feminino entre 17 e 49 anos, em que se avaliou a localização e diâmetro do OEM, além de histórico de tratamento cirúrgico para endometriose, os sintomas inespecíficos como, dismenorréia, dispareunia e dor pélvica crônica bem como, sintomas específicos, por exemplo, hematoquezia, dor intestinal, frequência e urgência urinária e hidronefrose. Percebeu-se então que 272 (89,5\%) pacientes tinham dismenorréia, 39 (12,8\%) tinham dispareunia e 17 (5,6\%) dor pélvica crônica, além de que, 73 (24\%) pacientes relataram tenesmo retal, 13 (4,3\%) dificuldade de defecação, 11(3,6\%) tinham dor a defecação, 6 (2\%) queixavam-se de diarreia e 8 (2,6\%) de hematoquezia. Já em relação aos sintomas urinários, 9 (3\%) pacientes relataram aumento da frequência e urgência urinária, $2(0,7 \%)$ queixavam de hematúria e $26(8,6 \%)$ apresentavam hidronefrose. Ao fazer a análise da localização observou-se que 98 (32,2\%) pacientes tinham OEM à esquerda, 86 (28,3\%) à direita e 120 (39,5\%) OEM bilateral com tamanho médio de 55,9 $\pm 23,9$ mm. Ademais, a avaliação do número médio de lesões de DIE por paciente foi de 2,60 $\pm 1,46$ totalizando 788 lesões confirmadas histologicamente, sendo que um total de 532 lesões foram registradas, das quais 274 (51,5\%) foram localizados à esquerda e $258(48,5 \%)$ à direita. Além disso, a distribuição anatômica das lesões de DIE foram as seguintes: 245 (80,6\%) lesões no ligamento uterosacro, 93 (30,6\%) no intestino, $47(15,5 \%)$ no ureter, 40 (13,7\%) na vagina, $33(10,9 \%)$ no fornix posterior, 29 $(9,54 \%)$ nas tubas uterinas, $21(6,91 \%)$ no reto vaginal, $9(2,96)$ na bolsa reto vaginal, $4(1,32 \%)$ na bexiga. Todavia, o presente estudo teve 4 casos de endometriose no apêndice que não foram incluídas na intestinal. Com isso, percebeu-se que OEM unilateral ( $\mathrm{n}=184$ pacientes) teve um número significativamente maior de lesões DIE quando comparados com pacientes com OEM bilateral, bem como uma maior taxa de infiltração intestinal $(\mathrm{P}<0,01)$ e vaginal $(\mathrm{P}<0,01)$ por DIE. Entretanto o número médio de lesões DIE não estava significativamente correlacionado com a localização $(\mathrm{P}=0,678)$ ou tamanho $(\mathrm{P}=0,518)$ do $\mathrm{OEM}$ e não teve diferença significativa entre os grupos com OEM $\leq 50 \mathrm{~mm}$ e $>50 \mathrm{~mm}$. Assim, concluiu-se que a distribuição das lesões de DIE associadas a OEM era, frequentemente, multifocal e severa.

Na pesquisa de Xiaopei Chao et al. (2020), buscaram fornecer uma referência para a prevenção, diagnóstico e tratamento de massas pélvicas através da análise clínicopatológicas em pacientes que passaram por histerectomia por doenças benignas, como endometriose e adenomiose. Para isso foi elaborado o delineamento de janeiro a dezembro de 2016, em um estudo observacional, com 247 mulheres de 22 a 59 anos, que foram subdivididas em dois grupos, em que o grupo A as pacientes tinham endometriose/adenomiose e no grupo B sem endometriose/adenomiose. Fez parte do projeto aquelas que tinham feito histerectomia em virtude de doença benigna, com reoperação devido à massa pélvica, levando-se em consideração as idades de histerectomia ( $<44$ anos $x \geq 44$ anos), suas idades de operação para massa pélvica ( $<51$ anos $x \geq 51$ anos), e o intervalo de tempo entre a histerectomia e o início da massa pélvica. Realizando a análise dos dados tem-se que $85,43 \%$ $(\mathrm{n}=211)$ das pacientes foram submetidas à histerectomia total, $14,57 \%(\mathrm{n}=36)$ histerectomia subtotal, sendo que $80,16 \%$ $(\mathrm{n}=198)$ das pacientes receberam histerectomia simples, 5,67\% $(\mathrm{n}=14)$ histerectomia e salpingectomia bilateral, 12,96\% $(\mathrm{n}=32)$ histerectomia e salpingooforectomia unilateral e $1,21 \%(n=3)$ histerectomia e salpingooforectomia bilateral. Do total de pacientes do estudo $23,08 \%(n=57)$ apresentavam adenomiose, enquanto que $12,55 \%(n=31)$ tinham endometriose e a idade 
média da cirurgia para retirada da massa pélvica foi de 50 anos, com intervalo de tempo médio entre a histerectomia e o início da massa pélvica de 5 anos. Além disso, 52,63\% dos casos teve a massa pélvica identificada incidentalmente pelo exame físico, 23,08\% tinham dores abdominais e 16,59\% queixavam de distensão abdominal e anorexia, enquanto que $28,74 \%$ das pacientes tinham soro elevado de CA 125 . Ademais, 34,01\% $(\mathrm{n}=84)$ foram diagnosticadas com tumores malignos, dos quais $76,19 \%$ ( $n=64$ ) eram ovarianos e o carcinoma epitelial ovariano representou $82,81 \%(n=53)$, enquanto que $65,99 \%$ ( $n=163$ ) do total de pacientes foram diagnosticados com tumores benignos, dos quais $67,48 \%$ surgiram do ovário, 19,02\% das tubas uterinas e a inflamação representou $12,27 \%$. Assim, obteve-se como resultado que o grupo A era mais jovem do que o grupo $\mathrm{B}$, o intervalo de tempo entre a histerectomia e o início da massa pélvica foi menor no grupo A, bem como o intervalo de tempo entre a histerectomia e a massa pélvica maligna confirmada também foi menor no grupo A, além de que a massa pélvica tendia a ser endometriose ovariana, cistos ovarianos fisiológicos e efusão pélvica encapsulada no grupo A. Todavia, a incidência de cistoadenoma seroso ovariano foi menor no grupo A $(\mathrm{P}=0,043)$ e esse mesmo grupo tinha um maior risco de carcinoma endometrioide $(P=0,095)$ e carcinoma de células claras do ovário $(P=0,065)$, enquanto o carcinoma seroso ovariano foi menor no grupo $\mathrm{A}(\mathrm{P}=0,129)$, mas sem diferença significativa. Além disso, não teve diferença significativa entre os dois grupos em pacientes que fizeram ressecção simples do útero com ou sem salpingooforectomia unilateral, mas o risco de massa pélvica maligna era maior no grupo A para aqueles que realizaram histerectomia com salpingectomia bilateral. Conclui-se então que a incidência de carcinoma endometrioide ovariano e carcinoma de células claras nos ovários eram maiores naqueles com um histórico de histerectomia devido a endometriose/adenomiose, assim como uma terapia adjuvante pós-histerectomia em virtude de doenças benignas pode contribuir com a prevenção de massa pélvica benigna.

Segundo projeto de Roxana-Cleopatra Penciu et al. (2020), buscou-se determinar se há alguma associação entre endometriose ovariana e câncer de ovário concomitantes, assim como determinar se há diferenças entre endometrioses associadas com diferentes tipos morfológicos de câncer de ovário. Para isso, rastrearam-se todos os pacientes - no Hospital Emergencial do Condado de Sf. Apostol Andrei, Constanta, Romênia - cirurgicamente tratados e patologicamente confirmados com endometriose entre 2015-2017 e os confirmados com câncer de ovário entre 2013-2017. Obteve-se 135 pacientes, dos quais somente 4 eram identificados concomitantemente para a endometriose e para o câncer de ovário. Como resultado, Dos 135 pacientes: 70 possuíam câncer de ovário ( 8 em estágio 1 e 62 em estágio de 2 a 4); 61 pacientes possuíam endometriose (40 em estágio 1, 13 em estágio 2 e 8 em estágio 3); 4 possuíam as duas doenças concomitantes (2 casos do tipo endometrioide e 2 casos do tipo câncer de ovário seroso de alto grau; todos esses quatro casos com endometriose em grau 1. Além disso, pacientes com endometriose e câncer de ovário do tipo endometrioide eram mais jovens e foram diagnosticados com estágios mais precoces de câncer; pacientes com endometriose e câncer de ovário do tipo seroso eram mais velhos e foram diagnosticados com estágios mais avançados de câncer. Somado a isso, a maioria dos casos de endometriose associada ao câncer de ovário (EAOCs) eram unilaterais. Já os cânceres de ovário não associados à endometriose eram, usualmente, bilaterais e associados à ascite. Em suma, o estudo não pode observar uma frequente associação entre endometriose e câncer de ovário do tipo endometrioide; não se encontrou qualquer similaridade entre endometriose e câncer de ovário sobre um ponto de visto morfológico e notou-se altos níveis de expressão de receptores de estrogênio para ambos os componentes - epitélio e estroma - em endometriose (EM) associada à câncer de ovário seroso de alto grau (HGSOC), quando comparado com EM associada ao tipo endometrioide.

No trabalho de Ota et al. (2020), foi relatado um caso de carcinoma endometrioide decorrente de lesões endometrioides infiltrativa profunda (EDI) no ligamento uterossacro 6 anos após o tratamento de malignidade ovariana limítrofe. Este é um caso raro de carcinoma relacionado à endometriose metacrônica, a paciente trata-se de uma mulher de 48 anos de etnia japonesa com histórico de 2 gravidez com 2 partos vaginais que apresentou histórico de dor pélvica intensa. Dentre os antecedentes a paciente tinha 13 anos na menarca, apresentava um ciclo menstrual regular e dismenorréia; não tinha 
histórico prévio de uso de terapia hormonal, incluindo contraceptivos orais. Com a pesquisa do quadro foi realizada ressonância magnética (RM) que revelou um tumor ovariano sólido e cístico de $4,4 \mathrm{~cm}$ de diâmetro no ovário direito com realce significativo pelo gadolínio e um cisto endometrial de 3,6 cm de diâmetro no ovário esquerdo. Os níveis dos marcadores tumorais, antígeno de carboidratos 19-9 (CA19-9) e CA-125, foram de $57 \mathrm{U} / \mathrm{ml}$ (referência < 37,0 U/mL) e $66 \mathrm{U} / \mathrm{ml}$ (referência $<35,0 \mathrm{U} / \mathrm{mL}$ ), respectivamente. Em razão dos resultados encontrados a paciente foi submetida à salpingoooforectomia bilateral laparoscópica (BSO), os achados laparoscópicos mostraram a presença de tumores ovarianos bilaterais com aderências graves ao redor do saco de Douglas devido ao desenvolvimento de endometriose. Após a BSO laparoscópica, uma lesão endometriótica residual suspeita de ser uma lesão de EDI foi detectada no ligamento útero-sacro esquerdo. O diagnóstico patológico dos ovários direito e esquerdo foi tumor endometrioide proliferativo atípico e endometrioma ovariano, respectivamente. Em consenso com a indicação médica e desejo da paciente foi feito o acompanhamento do tumor do ovário direito com tomografia computadorizada (TC) com contraste, foi realizada a cada 6 meses por 5 anos, sem recorrência da doença. Seis anos após a operação, foi identificada ascite na cavidade pélvica juntamente com um tumor sólido, localizado no lado esquerdo do saco de Douglas. A paciente foi submetida a uma PET/CT que revelou um acúmulo anormal de FDG no local do tumor. Os níveis de marcadores tumorais CA19-9 e CA-125 foram 81 U/ml (referência < 37,0 U/mL) e 45 U/ml (referência $<35,0 \mathrm{U} / \mathrm{mL}$ ), respectivamente. Portanto, suspeitava-se de tumor ovariano recorrente com malignidade limítrofe sendo realizada laparoscopia diagnóstica com achados que mostraram tumor no saco de Douglas enterrado e firmemente preso ao cólon sigmoide sem ascite aparente ou disseminação peritoneal. O tumor foi encontrado no local das lesões de EDI que estavam presentes 6 anos antes da cirurgia laparoscópica inicial. O tumor foi removido por cirurgia laparoscópica sendo realizado também histerectomia, omentectomia parcial e linfadenectomia pélvica e para-aórtica retroperitoneal por laparotomia. Posteriormente, foi feito um diagnóstico patológico de carcinoma endometrioide decorrente das lesões de EDI. A citologia do lavado peritoneal foi negativa e nenhuma metástase linfonodal foi identificada. O diagnóstico final foi um carcinoma peritoneal primário de estágio IIB baseado no sistema de estadiamento da Federação Internacional de Ginecologia e Obstetrícia. A paciente recebeu seis ciclos mensais de paclitaxel e carboplatina como quimioterapia adjuvante para seu câncer peritoneal. Não houve evidência de recorrência por 2 anos após o tratamento.

Em análise proposta por Okamoto et al. (2020) foi utilizado a hibridização genômica comparativa de alta resolução (CGH) para identificar alterações no número de cópias somáticas (SCNAs) associadas a cada uma das características clínicas de adenocarcinoma de células claras do ovário (CCC). As características clínicas do CCC incluem: 1) uma maior incidência entre japonesas, 2) uma associação com endometriose, 3) mau prognóstico em estágios avançados e 4) uma maior incidência de trombose como complicação. Para a análise das alterações foi utilizado o The Human Genome CGH 244A Oligo Microarray para examinar 144 amostras obtidas de 120 pacientes japoneses, 15 coreanos e 9 alemães com CCC. A mediana do período de acompanhamento foi de 24 meses (intervalo de 2 a 191 meses). A média de idade foi de 53 anos (intervalo; 30-86 anos). As etapas da FIGO foram as seguintes: Estágio I, 85; estágio II, 17; estágio III 39; estágio IV; 3. Dos 144 pacientes, 85 (71\%) foram considerados sensíveis à quimioterapia, 12 (10\%) foram intermediários e $22(19 \%)$ foram resistentes. Do total de pacientes, $43(30 \%)$ recidivaram e $29(20 \%)$ morreram. A endometriose foi diagnosticada em 68 (57\%) das pacientes japonesas, $6(40 \%)$ das coreanas e $2(22 \%)$ das pacientes alemãs; essas diferentes prevalências da endometriose foram estatisticamente significativas entre as populações comparadas $(\mathrm{P}=0,041)$. Foram analisados os valores-q dos SCANs associadas às características clínicas da CCC. Foi identificado que os pacientes com endometriose e aqueles sem diferiram significativamente em relação a 135 SCNAs, e o locus que inclui a amplificação do gene EGFR foi mais proeminente. Com base nos dados de CGH e na validação de dados de Q-PCR de amostras individuais e alta concordância (86\%) e altos valores de kappa (70\%), a prevalência de endometriose foi significativamente maior em pacientes com um locus amplificado do EGFR (85\%) do que naquelas sem amplificação do EGFR (35\%) (P<0,0001). Além disso, com base na análise de RT-PCR, os níveis 
de RNA do EGFR foram significativamente maiores em amostras de pacientes com endometriose do que naqueles sem ela ( $\mathrm{P}$ $=0,037)$. Em contraste com os SCNAs, não foram observadas mutações patológicas do EGFR (dados não mostrados).

\section{Considerações Finais}

O objetivo da revisão foi identificar possíveis relações entre o câncer de ovário e a endometriose, pois acredita-se haver concomitância entre as duas patologias. No entanto, concluiu-se, nessa revisão integrativa, que não há relação significativa entre elas, devido às baixas porcentagens encontradas. Além disso, houve maior conexão entre a endometriose e alguns tipos histológicos de câncer de ovário, assim como uma maior incidência em determinadas localidades, como o Japão.

Ademais, as evidências até então têm como função a melhora no diagnóstico de câncer de ovário, assim como o prognóstico de cada subtipo, possibilitando os possíveis tratamentos em estágios iniciais do tumor, o que aumenta as chances de recuperação e sobrevivência do paciente.

É crucial, portanto, haver maior investigação, com base em critérios mais padronizados, com maior quantidade amostral, bem como um maior número de pesquisas a níveis moleculares - visto que foram encontradas relações promissoras no âmbito molecular.

\section{Referências}

Acién, P., Velasco, I., Acién. M., Capello, C. \& Vela, P. (2015). Epithelial ovarian cancers and endometriosis. Gynecologic and obstetric investigation, 79(2),126-135. https://doi.org/ 10.1159/000367597.

Bellelis, P., Jr, J. A. D., Podgaec, S., Gonzales, M., Baracat, E. C. \& Abrão, M.S. (2010). Aspectos epidemiológicos e clínicos da endometriose pélvica - uma série de casos. Revista da associação médica brasileira, 56(4), 467-471. https://doi.org/10.1590/S0104-42302010000400022.

Chao, X., Liu, Y., Ji, M., Wang, S., Shi, H., Fan, Q. \& Lang, J. (2020). Malignant risk of pelvic mass after hysterectomy for adenomyosis or endometriosis. Medicine, 99(15). https://doi.org/10.1097/MD.0000000000019712.

Sousa, L. D., Lunardi F, W. D., Lunardi, V. L., Santos, S. S. C. \& Dos Santos, C. P. (2011). The nursing scientific production about the clinic: an integrative review. Rev Esc Enferm, 45 (2), 494-500. https://doi.org/10.1590/S0080-62342011000200027.

Galvao,C. M., Sawada,N. O. \& Trevizan,M. A. (2004). Systematic review: a resource that allows the incorporation of evidence into nursing practice. Revista Latino-americana Enfermagem, 17 (4), 758-764. https://doi.org/10.1590/S0104-11692004000300014 .

Jimenez, J. C. V., Muñoz, E. V., Poveda, I. G., Lorente, D. S., Pérez, B. S. \& Lopez, J. S. J. (2019). Diagnostic challenges: low-grade adenosarcoma on deep endometriosis. BMC Womens Health, 19(1): 124. https://doi.org/10.1186/s12905-019-0815-1.

Kwok, H., Jiang, H., Li, T., Yang, H., Fei, H., Cheng, L., Yao, S. \& Chen, S. (2020). Lesion distribution characteristics of deep infiltrating endometriosis with ovarian endometrioma: an observational clinical study. BMC women's health, 20(1), 111. https://doi.org/10.1186/s12905-020-00974-y.

Lee, W. L., Chang, W. H., Wang, K. C., Guo, C. Y., Chou, Y. J., Huang, N., Huang, H. Y., Yen, M. S., Wang, P. H. (2015). The Risk of Epithelial Ovarian Cancer of Women With Endometriosis May be Varied Greatly if Diagnostic Criteria Are Different: A Nationwide Population-Based Cohort Study. Medicine, 94(39). https://doi.org/10.1097/MD.0000000000001633.

Marí-Alexandra, J., Cacelén, A. P., Agababyan. C., Moreno-ManueL, M., García-Oms, J., Calabuig-Fariñas, S. \& Gilabert-Estellés, J.(2019). Interplay Between MicroRNAs and Oxidative Stress in Ovarian Conditions with a Focus on Ovarian Cancer and Endometriosis. International Journal Of Molecular Sciences, 20(21), 5322-5325. http://dx.doi.org/10.3390/ijms20215322.

Meng, Q., Sun, W., Jiang, J., M Fletcher, N., Diamond, M.P. \& Saed, G.M. (2011). Identification of common mechanisms between endometriosis and ovarian cancer. Journal of Assisted Reproduction and genetics, 28(10), 917-923. https://doi.org/10.1007/s10815-011-9573-1.

Mikhaleva, L. M., Davydov, A. I., Patsap, O. I., Mikhaylenko, E. V., Nikolenko,V. N., Neganova, M. E., Klochkov, S. G., Somasundaram, S. G., Kirkland, C.E. \& Aliev, G.(2020). Malignant Transformation and Associated Biomarkers of Ovarian Endometriosis: a narrative review. Advances In Therapy, 37(6), 2580-2603. https://doi.org/10.1007/s12325-020-01363-5.

Murakami, K., Kotani, Y., Nakai, H. \& Matsumura, N. (2020) Endometriosis-Associated Ovarian Cancer: the origin and targeted therapy. Cancers, 12(6),1676. http://dx.doi.org/10.3390/cancers12061676.

Nezhat, C., Vu, M., Vang, N., Ganjoo, K., Karam, A., Folkins, A., Nezhat, A. \& Nezhat, F. (2019). Endometriosis Malignant Transformation Review: Rhabdomyosarcoma Arising From an Endometrioma. JSLS:Journal of the Society of Laparoendoscopic Surgeons,23(4). https://doi.org/10.4293/JSLS.2019.00038.

Okamoto, A., Sehouli, J., YAnaihara, N., Hirata, Y., Braicu, I., Kim, B.G., Takakura, S., Saito, M., Yanagida, S., Takenaka, M., Yamaguchi, N., Morikawa, A., Tanabe, H., Yamada, K., Yoshihara, K., Enomoto, T., Itamochi, H., Kigawa, J., Matsumura, N., Konishi, I., Aida, S., Aoki, Y., Ishii, N., Ochiai, 
K., Akiyama, T. \& Urashima, M. (2020). Somatic copy number alterations associated with Japanese or endometriosis in ovarian clear cell adenocarcinoma. Plos One, 10(2). https://10.1371/journal.pone.0116977.

Oliveira, R., Musich, D. D. S., Ferreira, M. P. S. F., Vilarino, F.L. \& Barbosa, C.P. (2015). Perfil epidemiológico das pacientes inférteis com endometriose. Reprodução \& Climatério, 30(1), 5-10. http://dx.doi.org/10.1016/j.recli.2015.03.005.

Olmos, M. L., Peña, A. C. R.., Martínez, M. J. P., Asturiano, M.E.L., García, C.F., Sáenz, L.S.J. \& Campos, M.G. (2020). Carcinoma endometrioide sincrónico de ovario y endometrio. Revista Chilena d Obstreticia y Ginecología, 85(3), 263-269. http://dx.doi.org/10.4067/s0717-75262020000300263.

Ota, Y., Ota, K., Takahashi, T., Suzki, S., Sano,R., Ota, I., Moriya, T. \& Shiota, M. (2020). Primary endometrioid carcinoma of the uterosacral ligament arising from deep infiltrating endometriosis 6 years after bilateral salpingo-oophorectomy due to atypical proliferative endometrioid tumor of the ovary: a rare case report. World journal of surgical oncology, 18(1), 329. https://doi.org/10.1186/s12957-020-02105-1.

Pearce, C. L., Templeman, C., Rossing, M. A. et al. (2012). Association between endometriosis and risk of histological subtypes of ovarian cancer: a pooled analysis of case-control studies. Lancet Oncology, 13(4), 385-394. https://doi.org/10.1016/S1470-2045(11)70404-1

Penciu, R. C., Postolache, I., Steriu, L., Izavoranu, S., Tica, A. A., Mocanu, I. D., Sârbu, V., Deacu, M., Tica, I., Bălţătescu, G. I., Tica, O. S. \& Tica, V. I. (2020). Is there a relationship in-between ovarian endometriosis and ovarian cancer? Immunohistochemical profile of four cases with coexisting ovarian endometriosis and cancer. Romanian journal of morphology and embryology, 61(1), 157-165. https://doi.org/10.47162/RJME.61.1.18

Salomé, D. G. M., Braga, A. C. B. P., Lara, T. M. \& Caetano, O.A. (2020). Endometriose: epidemiologia nacional dos últimos 5 anos. Revista de Saúde, 11(2), 39-43. https://doi.org/10.21727/rs.v11i1.2427

Sevilla, I. N., Linde, F. M., Sánchez, M. M., Arense, J. J., Torroba, A., Díaz, A. N. \& Ferrer, M. L. S. (2019). Prognostic importance of atypical endometriosis with architectural hyperplasia versus cytologic atypia in endometriosis-associated ovarian cancer. Journal of gynecologic oncology, 30(4). https://doi.org/10.3802/jgo.2019.30.e63

Suryawanshi, S., Vlad, A. M., Lin, H., Smaldone,G.M., Laskey, R., Lee, M., Lin, Y., Donnellan, N., Patel, M.K., Lee,T., Mansuria,S., Elishaev, E., Budiu, R., Edwards, R.P. \& Huang, X. (2013). Plasma microRNAs as novel biomarkers for endometriosis and endometriosis-associated ovarian cancer. Clinical Cancer Research, 19(5), 1213-1224. https://doi.org/10.1158/1078-0432.ccr-12-2726

Xiao, W., Awadallah, A. \& Xin, W. (2012). Loss of ARID1A/BAF250a expression in ovarian endometriosis and clear cell carcinoma. International journal of clinical and experimental pathology, 5(7), 642-650. https://pubmed.ncbi.nlm.nih.gov/22977660/.

Whittemore R \& Knafl K. (2005). The integrative review: updated methodology. J Adv Nurs, 52 (5), 546-553. https://doi.org/10.1111/j.13652648.2005.03621.x.

Zhang, W., Jia, S., Xiang, Y., Yang, J., Jia, C. \& Leng, J. (2018). Comparative study of endometrioid borderline ovarian tumor with and without endometriosis. Journal of ovarian research, 11(1), 67. https://doi.org/10.1186/s13048-018-0440-x. 